\title{
S-nitrosylation of proteins at the leading edge of migrating trophoblasts by inducible nitric oxide synthase promotes trophoblast invasion ${ }^{\text {is }}$
}

\author{
Lynda K. Harris ${ }^{a}$, James McCormick ${ }^{b}$, Judith E. Cartwright ${ }^{b}$, \\ Guy St.J Whitley ${ }^{b}$, Philip R. Dash ${ }^{b, *}$ \\ ${ }^{a}$ Maternal and Fetal Health Research Centre, Division of Human Development, University of Manchester, St. Mary's Hospital, Manchester, UK \\ ${ }^{\mathrm{b}}$ Department of Basic Medical Sciences, St.George's, University of London, UK
}

\section{A R T I C L E I N F O R M A T I O N}

Article Chronology:

Received 5 October 2007

Revised version received

30 January 2008

Accepted 15 February 2008

Available online 29 February 2008

\section{Keywords:}

Nitrosylation

Trophoblast

Migration

Invasion

Nitric oxide

MMP

\begin{abstract}
A B S T R A C T
Nitric oxide regulates many important cellular processes including motility and invasion. Many of its effects are mediated through the modification of specific cysteine residues in target proteins, a process called S-nitrosylation. Here we show that S-nitrosylation of proteins occurs at the leading edge of migrating trophoblasts and can be attributed to the specific enrichment of inducible nitric oxide synthase (iNOS/NOS2) in this region. Localisation of iNOS to the leading edge is co-incidental with a site of extensive actin polymerisation and is only observed in actively migrating cells. In contrast endothelial nitric oxide synathse (eNOS/NOS3) shows distribution that is distinct and non-colocalised with iNOS, suggesting that the protein S-nitrosylation observed at the leading edge is caused only by iNOS and not eNOS. We have identified MMP- 9 as a potential target for S-nitrosylation in these cells and demonstrate that it co-localises with iNOS at the leading edge of migrating cells. We further demonstrate that iNOS plays an important role in promoting trophoblast invasion, which is an essential process in the establishment of a successful pregnancy.
\end{abstract}

(c) 2008 Elsevier Inc. All rights reserved.

\section{Introduction}

Nitric oxide (NO) is an important signalling molecule that is involved in regulating a diverse range of cellular processes such as apoptosis, cell division and cell differentiation. The effects of NO can be mediated through the production of cyclic GMP folowing its reaction with the haem group of guanylate cyclase or through the modification of either tyrosine or cysteine residues in proteins. The modification of cysteine groups by NO, a process known as S-nitrosylation, is being increasingly recognised as an important post-translational protein modification with many similarities to phosphorylation [1,2]. Protein S-nitrosylation has been shown to be able to either activate or inactivate protein function, depending on the protein that is modified. It exhibits strict spatial and temporal regulations and is reversible [3]. Over a hundred proteins have been identified as being nitrosylated [4] and the regulation of this process, particularly how its specificity is achieved, is an important area of investigation.

Trophoblasts are one of the earliest cell types to differentiate from the mammalian embryo and are responsible for the formation of the placenta. Trophoblasts, in particular the extravillous sub-population, are highly motile and invasive cells.

This work was supported by the British Heart Foundation (Grant Number FS/03/053/15869) and the Wellcome Trust (Grant Number 069939).

* Corresponding author.

E-mail address: p.r.dash@reading.ac.uk (P.R. Dash). 
During early pregnancy they invade the uterine wall and remodel the maternal arteries, facilitating delivery of a high flow, low resistance blood supply to the developing fetus [5]. Trophoblasts constitutively express two isoforms of nitric oxide synthase, the enzymes which produce NO in cells. These isoforms are eNOS and iNOS [6-8]. We have previously shown that NO plays an important role in regulating apoptosis [8-10] and cell motility [11,12] in trophoblasts.

Trophoblast migration and invasion are critical processes in the establishment of a successful pregnancy. Poor trophoblast invasion has been associated with the development of diseases of pregnancy such as pre-eclampsia and intrauterine growth restriction (IUGR) [13] and an understanding of the regulation of this process is therefore of considerable interest. Cell migration and invasion are complex and tightly regulated processes. The initial stages of migration involve polarisation of the cell followed by protrusion of the plasma membrane from the leading edge of the cell, sometimes referred to as the lamellipodium. Adhesion of the plasma membrane protrusions then occurs, which is followed by retraction of the trailing edge. This process is then repeated to allow forward movement of the cell. In addition cells must also degrade the extracellular matrix, typically using matrix metalloproteinases, in order to produce space through which the cell can move.

Nitric oxide has been shown to affect cell migration and invasion in a wide variety of cell types [14-16], including trophoblasts [11]. Here we report that the inducible isoform of nitric oxide synthase, iNOS, is specifically enriched at the leading edge of migrating trophoblast cells. We also show that iNOS is responsible for the site specific S-nitrosylation of proteins at the leading edge and identify a possible role of NO
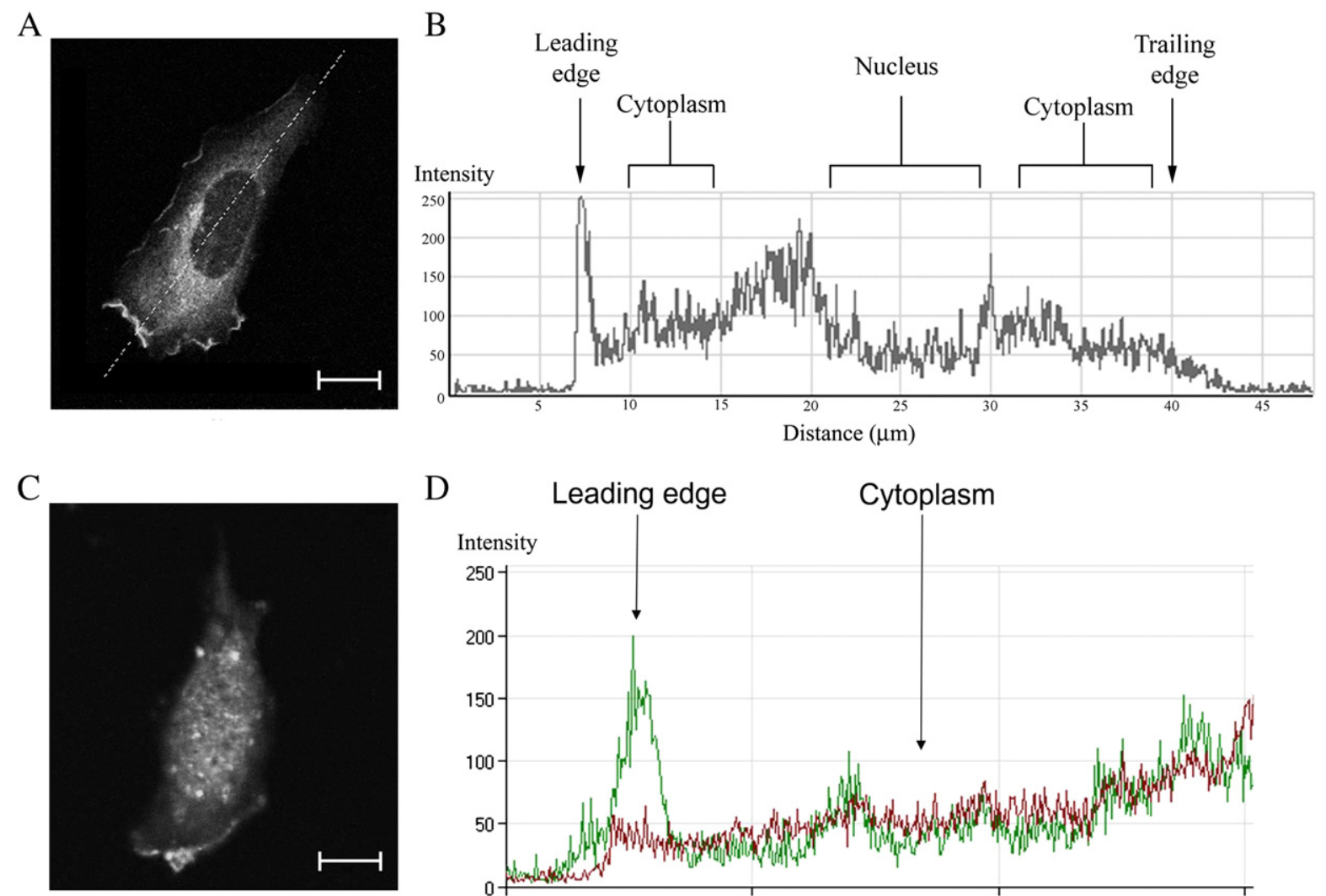

$\mathrm{D}$

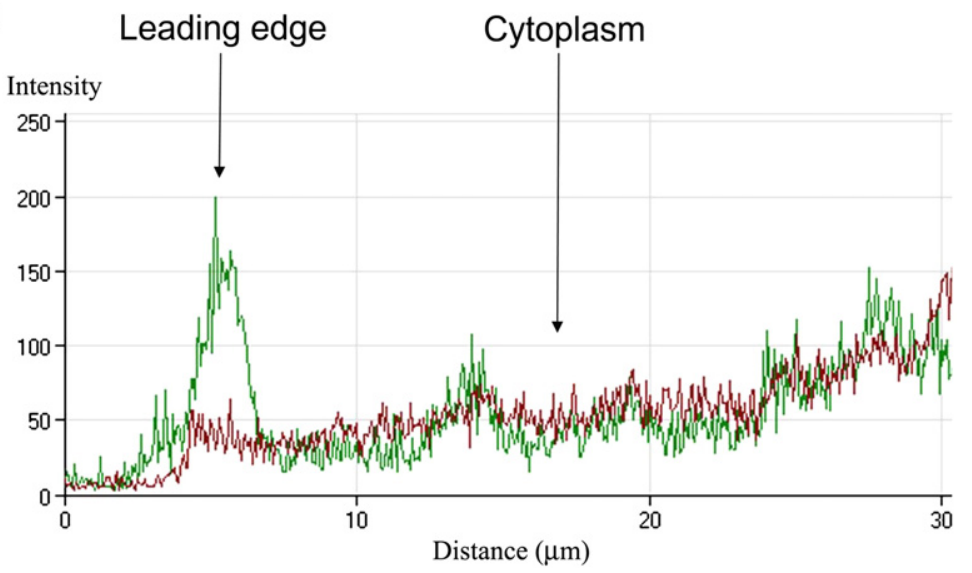

Fig. 1 - Localisation of iNOS at the leading edge of migrating trophoblast cells. (A) Intracellular distribution of iNOS-EGFP expressed in a migrating trophoblast cell. The image shown is a single z-section obtained by confocal microscopy. The scale bar represents $10 \mu \mathrm{m}$ and the dotted line represents the line that was quantified and shown in panel B. The cell is a representative of at least 50 cells observed over three separate experiments. (B) Fluorescence intensity of the dotted line shown in panel A showing increased iNOS localised at the leading edge. (C) Immunocytochemical analysis of the distribution of iNOS in migrating cells using an anti-iNOS antibody. The image shown is a single z-section obtained by confocal microscopy. The scale bar represents $10 \mu \mathrm{m}$. The cell is a representative of at least 50 cells observed over three separate experiments. (D) Quantification of the fluorescence intensity along a line drawn through a cell expressing iNOS-EGFP and labelled with CellTracker Orange dye. The green line represents the intensity of iNOS-EGFP, while the red line represents the intensity of the CellTracker dye. Increased intensity of iNOS at the leading edge relative to CellTracker intensity suggests that iNOS is specifically enriched at the leading edge. The quantification profile shown is a representative of at least 40 cells analysed in three separate experiments. 
in trophoblast invasion as MMP-9 co-localises with iNOS at the leading edge of these cells.

\section{Materials and methods}

\section{Materials}

Antibodies against iNOS and eNOS were obtained from Santa Cruz Biotechnology (Autogen Bioclear, UK), the MMP-9 polyclonal antibody was obtained from Cell Signalling Technology (New England Biolabs, UK) and the nitrosocysteine monoclonal antibody was obtained from AG Scientific (UK). Biotinylated secondary antibodies were obtained from Vector Labs (UK). Phalloidin-AlexaFluor546, CellTracker Orange, streptavidin-AlexaFluor488, streptavidin-AlexaFluor 350 and streptavidin-AlexaFluor546 were obtained from Molecular Probes (Invitrogen, UK). The NOS inhibitor L-NAME was obtained from Sigma (UK), the iNOS inhibitor 1400W was from Axxora (UK) and the Vectashield mounting medium containing DAPI was from Vector Labs (UK). The MMP-9 inhibitor $\mathrm{N}$-isobutyl-N-(4-methoxyphenylsulfonyl)-glycyl hydroxamic acid (NNGH) was from Biomol International (Exeter, UK). The iNOS-EGFP plasmid was constructed as previously described [15] and the iNOS-citrine plasmid was a kind gift from Dr. Joel Swanson, University of Michigan. Cells were transiently transfected using FuGENE 6 transfection reagent from Roche (UK). Matrigel ${ }^{\mathrm{TM}}$ was purchased from $\mathrm{BD}$ Discovery Labware (Bedford, MA, USA).

Cell culture

SGHPL-4 cells are derived from primary first trimester extravillous trophoblasts transfected with the early region of SV40. Characterization of these cells has confirmed an extravillous trophoblast-like phenotype including expression of HLA-G, cytokeratin-7, $[11,17,18]$. SGHPL-4 cells were cultured in Hams F10 media supplemented with glutamine (2 mM), penicillin (100 U/ml), streptomycin $(0.1 \mathrm{mg} / \mathrm{ml})$ and $10 \%(\mathrm{v} / \mathrm{v})$ fetal calf serum.

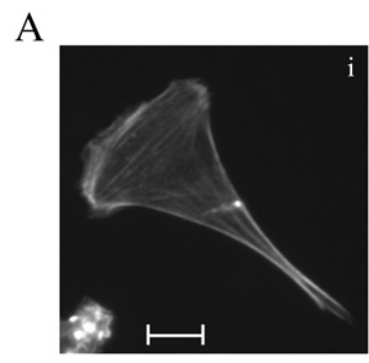

Actin

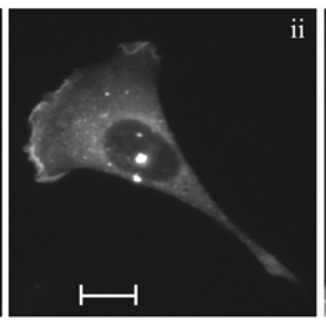

iNOS-GFP

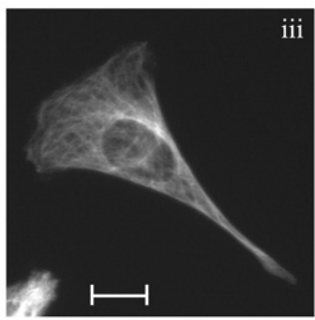

Tubulin

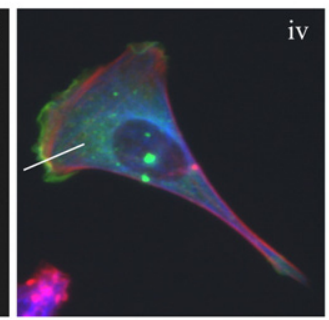

Merged

$\mathrm{B}$

Leading

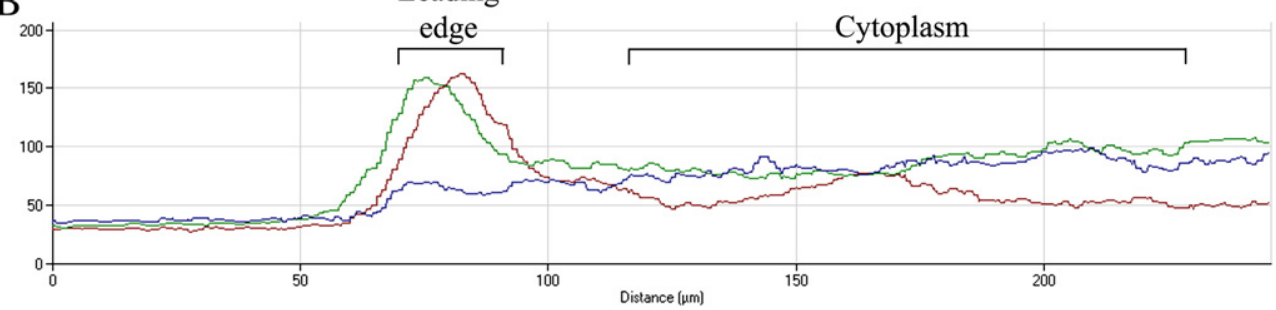

C
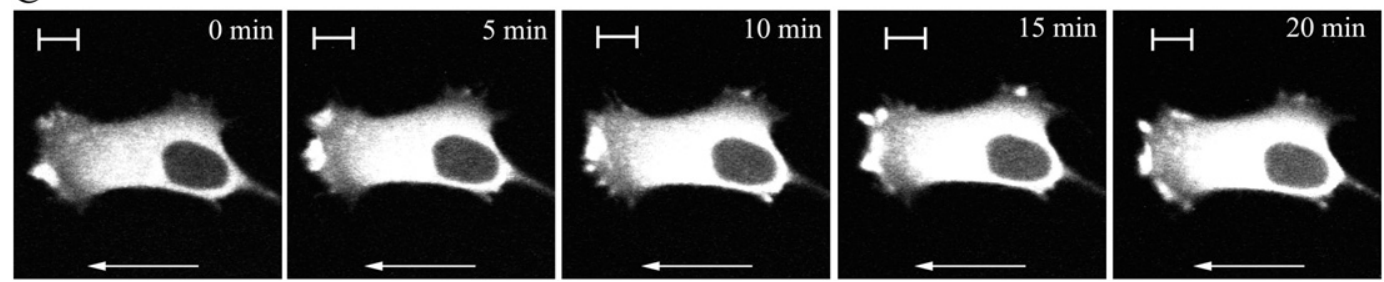

Fig. 2 - Localisation of iNOS to the site of actin polymerisation at the leading edge of migrating trophoblast cells.

(A) Fluorescence microscope image of a migrating trophoblast cell showing actin (stained with phalloidin-AlexaFluor546 and shown in red), anti-tubulin antibody (detected with anti-rabbit secondary antibody conjugated with AlexFluor350 and shown in blue) and iNOS-EGFP (shown in green). Image is a representative of at least 50 migrating cells from three separate experiments. The scale bar represents $10 \mu \mathrm{m}$. (B) Quantification of the fluorescence intensity along the line shown in panel A. The green line represents the intensity of iNOS-EGFP, the red line represents actin and the blue line represents tubulin staining. The quantification profile represents at least 20 cells from three separate experiments. (C) Time-lapse images from a cell expressing iNOS-citrine. Images were captured every 2 min over a 30 minute period. The arrow indicates the direction of movement of the cell and the scale bar represents $10 \mu \mathrm{m}$. 


\section{Isolation of primary extravillous trophoblasts}

Informed consent was obtained for all placental tissue used in this study and ethical committee approval was in place. Normal first trimester placenta was obtained at elective termination of pregnancy. Primary first trimester trophoblasts were cultured in a 1:1 ratio of DMEM:Ham's F12 culture medium supplemented with $10 \%$ FBS, L-glutamine (2 mM), penicillin $(100 \mathrm{IU} / \mathrm{ml})$ and streptomycin $(100 \mu \mathrm{g} / \mathrm{ml})$. Isolation of primary trophoblasts was performed as previously described $[19,20]$. Cell isolates were plated onto Matrigel ${ }^{\text {TM }}$ coated flasks for up to $48 \mathrm{~h}$ to promote a more advanced extravillous phe- notype. Cells were then stained for the presence of iNOS and MMP-9 using the immunocytochemistry protocol described below.

\section{Confocal microscopy}

Images were captured using a Zeiss LSM 510 META laser scanning microscope equipped with $\mathrm{HeNe}$, Ar and UV lasers. Unless otherwise stated imaging was performed using a PlanApochromat $63 \times / 1.4$ Oil objective in multi-track scan mode. Quantification of the images, including quantitative colocalisation analysis, was performed using the Zeiss LSM 5 software.
A

\section{DAPI}

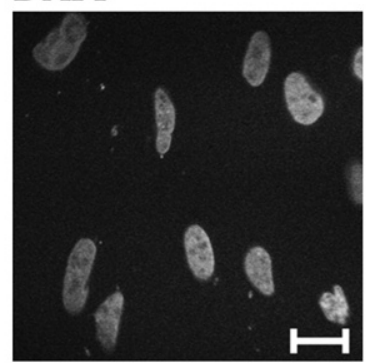

B

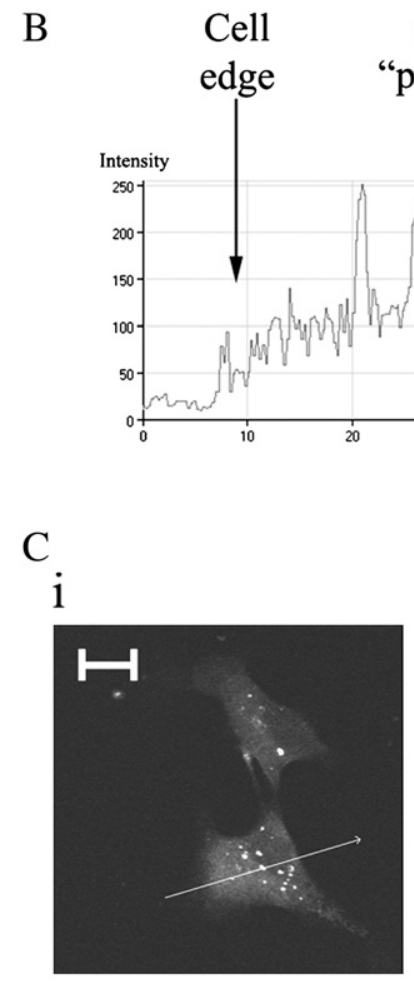

CellTracker Orange

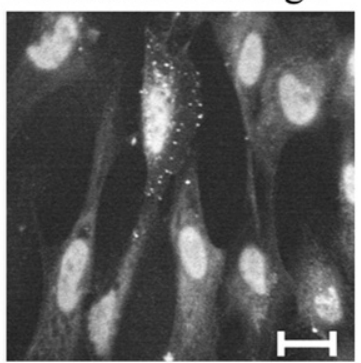

iNOS
iNOS-GFP

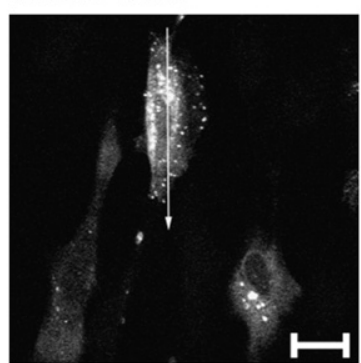

Cell

edge

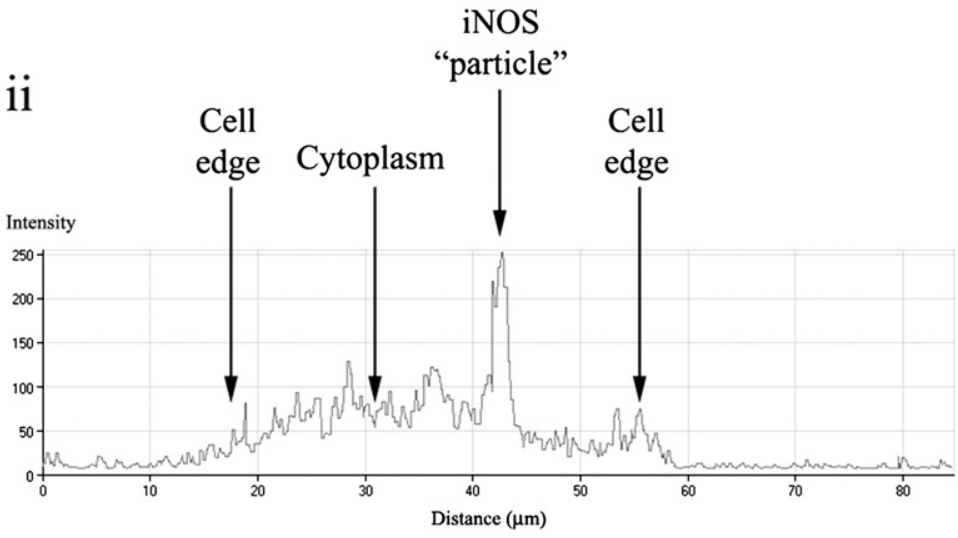

Fig. 3 - Distribution of iNOS in non-motile cells. (A) Confocal microscope images of confluent trophoblast cells expressing iNOS-EGFP and stained with the CellTracker Orange fluorescent protein dye. The image shown is a single z-section and the scale bar represents $20 \mu \mathrm{M}$. The image represents at least 30 fields of view from three separate experiments. The arrow indicates the line that was quantified for panel B. (B) Quantification of the fluorescence intensity of iNOS-EGFP along the line profile shown in panel A. Results are representative of at least 20 cells analysed from three separate experiments. (C) (i) Confocal microscope images of serum deprived trophoblast cells expressing iNOS-EGFP. The scale bar represents $20 \mu \mathrm{m}$ and the image represents at least 30 fields of view from three separate experiments. (ii) Quantification of the line represented by the arrow in (i). Results are representative of at least 30 cells analysed from three separate experiments. 
In some cases fluorescence microscopy was used to capture images. In these cases an Olympus IX71 inverted microscope equipped with a Hamamatsu C4742-95 digital camera was used. Images were captured using a $20 \times$ objective with an additional 1.6x adaptor. Migrating cells were defined as cells showing asymmetrical polarized morphology with one end of the cell, the leading edge, flatter and more spread out than the other end, often accompanied by the formation of membrane ruffles. Live cell phase-contrast time-lapse microcopy confirmed that this morphology was consistent with cell migration.

\section{Immunocytochemistry}

Cells were grown on gelatine-coated slides under standard tissue culture conditions. Cells were fixed in $2 \%$ paraformaldehyde in phosphate buffered saline (PBS) for $20 \mathrm{~min}$ at room temperature, then permeabilised with $0.2 \%$ Triton $\mathrm{X}-100$ in PBS for $15 \mathrm{~min}$. Samples were then washed with PBS and blocked for $1 \mathrm{~h}$ with 10\% goat serum in PBS. Primary antibodies were then added at a 1:100 dilution in $2 \%$ goat serum for $1 \mathrm{~h}$. Samples were then washed 3 times with PBS, for 5 min each time, before adding a biotinylated secondary antibody at a dilution of 1:100 for $1 \mathrm{~h}$. Samples were then washed a further 3 times with PBS before adding either streptavidin-AlexaFluor488, streptavidinAlexaFluor546 or streptavidin-AlexaFluor350 at a dilution of 1:66 in PBS for $30 \mathrm{~min}$. Samples were washed 3 times in PBS and mounted in Vectashield mounting media in the presence or absence of DAPI.

\section{Detection of S-nitrosylated proteins}

Immunostaining of S-nitrosylated proteins was performed using a specific anti-nitrosocysteine antibody as previously described [21]. Briefly, cells were plated on gelatine-coated slides at around 30\% confluency and allowed to attach for at least $16 \mathrm{~h}$. Cells were then fixed in $2 \%$ paraformaldehyde in PBS for 20 min at room temperature and then permeabilised with $0.2 \%$ Triton $\mathrm{X}-100$ in PBS for $15 \mathrm{~min}$. Cells were then blocked for $1 \mathrm{~h}$ using $10 \%$ goat serum containing $0.1 \mathrm{mM}$ neocuproine and $1 \mathrm{mM}$ EDTA and then cells were incubated with the monoclonal anti-nitrosocysteine antibody $\left(1 \mu \mathrm{g} / \mathrm{ml}\right.$ in $2 \%$ goat serum) overnight at $4{ }^{\circ} \mathrm{C}$. After washing, the cells were incubated with the secondary antibody, AlexaFluor546 conjugated anti-mouse (1:100), in $2 \%$ goat serum at room temperature for $1 \mathrm{~h}$. As an antibody control, cells were incubated with $1 \mu \mathrm{g} / \mathrm{ml}$ of mouse immunoglobulins in place of the primary antibody. In addition cells were treated with the nitric oxide synthase inhibitor, L-NAME ( $5 \mathrm{mM}$ ) for $24 \mathrm{~h}$ prior to immunostaining.

\section{Invasion assay}

Cells were plated onto $10 \mathrm{~mm}$ transwell inserts (Nunc, UK) with $8 \mu \mathrm{m}$ pores which had been coated with a thin layer of Matrigel (BD Biosciences, UK) and placed into wells in a 24-well plate. Cells were incubated at $37^{\circ} \mathrm{C}$ for $24 \mathrm{~h}$ in the presence or absence of either MMP inhibitors or NOS inhibitors. The culture medium was then aspirated and the transwells washed with PBS then fixed in $4 \%$ paraformaldehyde in PBS for $15 \mathrm{~min}$. The
A i

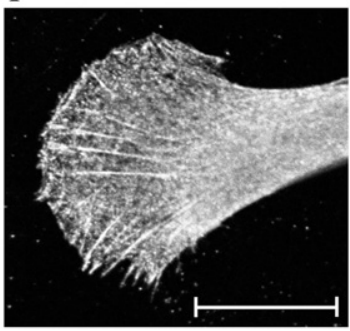

B

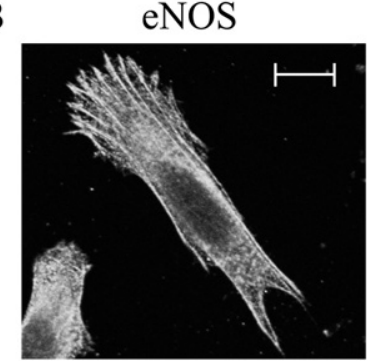

ii

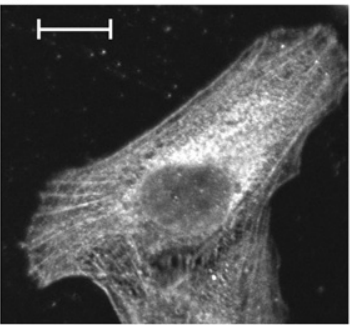

Actin

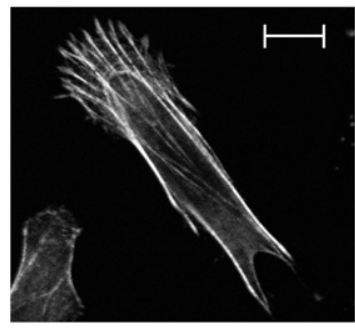

iii

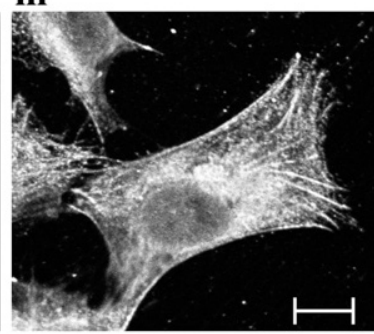

Merged

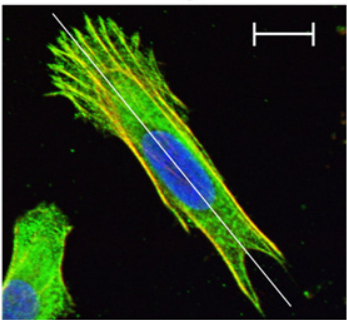

Fig. 4 - Distribution of eNOS in trophoblasts. (A) Confocal microscope image showing the intracellular distribution of eNOS in trophoblast cells as determined by immunocytochemistry using an anti-eNOS polyclonal antibody. The images shown are single $z$-sections and the scale bars represent $10 \mu \mathrm{m}$. The images shown are representative of at least 40 cells observed in three separate experiments. (i) Shows eNOS distribution in a migrating cell (ii) and (iii) shows eNOS distribution in non-migrating cells. (B) Confocal microscope image showing the intracellular distribution of eNOS in trophoblast cells as determined by immunocytochemistry. Cells were labelled with an anti-eNOS polyclonal antibody (shown in green), phalloidin-AlexaFluor 546 (shown in red) and DAPI (shown in blue). The image shown is a single z-section and the scale bar represents $10 \mu \mathrm{m}$. The image shown is a representative of at least $\mathbf{4 0}$ cells observed in three separate experiments. 
transwells were then removed from the plates and the upper side of each membrane was wiped with a cotton bud to remove the cells. The cells on the lower side of the inserts were then stained using Harris hematoxylin for $15 \mathrm{~min}$. The number of cells per field was counted using a $\times 20$ objective and the results expressed as the percentage of the total number of cells.

\section{Results}

iNOS is specifically enriched at the leading edge of migrating trophoblasts

Cells expressing an iNOS-EGFP fusion protein showed enhanced fluorescence at the leading edge of migrating cells. Fig. 1A shows a typical motile trophoblast cell with iNOS distributed in the cytoplasm and at the leading edge. The fluorescence intensity was quantified along the line and the results are shown in Fig. 1B. The highest fluorescence was detected at the leading edge, with relatively little iNOS immediately behind this region. This distribution pattern was confirmed when basal iNOS was detected by immunocytochemical staining (Fig. 1C). Staining of trophoblast cells with the CellTracker Orange stain, which labels proteins non-specifically, showed no corresponding increase in fluorescence at the leading edge (Fig. 1D), suggesting that iNOS was specifically enriched at the leading edge.

iNOS distributes to the site of increased actin polymerisation and is rapidly re-distributed in living cells

Increased actin polymerisation at the leading edge is a key feature of cell movement and the enhanced distribution of iNOS at the leading edge was found to be co-incident with increased actin staining (Fig. 2A). In some cases iNOS colocalised with the site of actin polymerisation but in many cases it appeared that the iNOS was located slightly in advance of the actin polymerisation (Fig. 2B). The distribution of iNOS-citrine in live cells was observed using time-lapse confocal microscopy. iNOS was rapidly re-distributed around the leading edge (Fig. 2C) suggesting active transport of iNOS around this region.

iNOS is not membrane associated in non-motile cells

The distribution of iNOS in non-motile cells was compared to that of motile cells. In confluent cells iNOS was detected in the cytoplasm and in particulate form, but none was detected at the plasma membrane (Fig. 3A and B). Motility was also reduced through serum starvation for $24 \mathrm{~h}$ (Fig. 3C) and again it
A
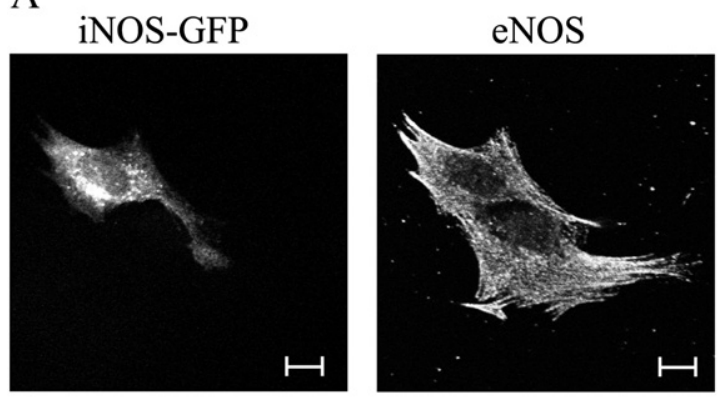

C

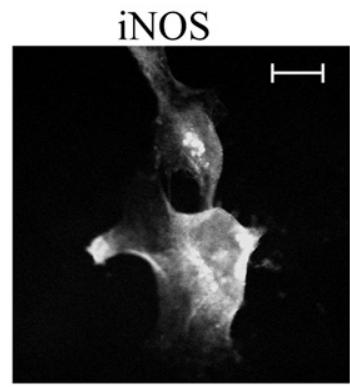

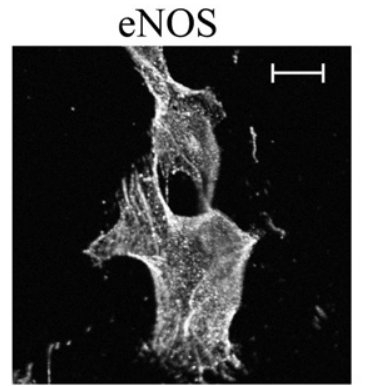

B

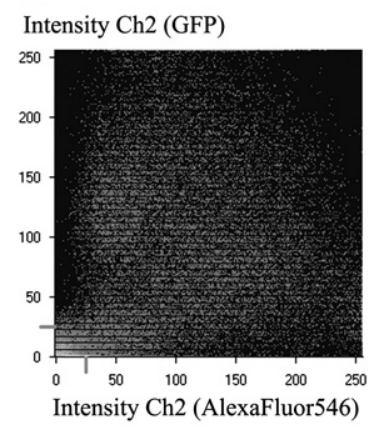

$\mathrm{D}$

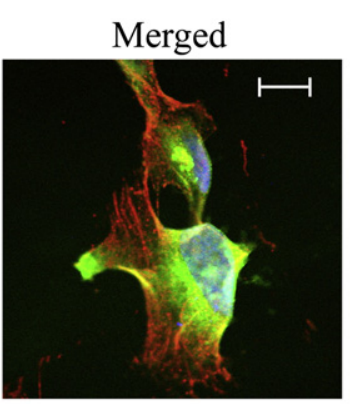

Intensity $\mathrm{Ch} 2$

(AlexaFluor488)

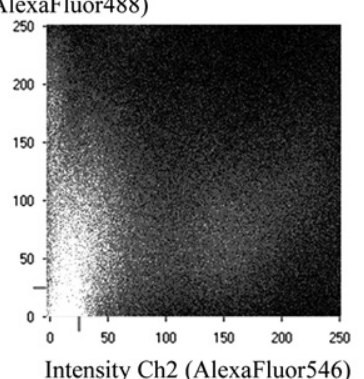

Fig. 5 - Distribution of eNOS and iNOS in the same cells. (A) Confocal microscope images showing the distribution of eNOS (shown in red), iNOS-EGFP (shown in green) and DAPI (shown in blue) in trophoblasts. The image shown is a single z-section and the scale bar represents $10 \mu \mathrm{m}$. The image is a representative of at least 30 cells from three separate experiments.

(B) Quantitative co-localisation analysis of iNOS-GFP expressing cells labelled for eNOS using immunocytochemistry. The figure is a representative of co-localisation analysis in at least 20 cells. (C) Confocal microscope images showing the distribution of eNOS (shown in red), iNOS (shown in green) and DAPI (shown in blue) in trophoblasts. Both eNOS and iNOS were detected using isoform specific antibodies. The image shown is a single $z$-section and the scale bar represents $10 \mu \mathrm{m}$. The image is a representative of at least 30 cells from three separate experiments. (D) Co-localisation analysis of cells labelled for both iNOS and eNOS using immunocytochemistry. The figure is a representative of co-localisation analysis in at least 20 cells. 
was found that there was no iNOS associated with the plasma membrane, suggesting that iNOS is only translocated to the plasma membrane during cell migration.

eNOS is distributed in the cytoplasm and along actin filaments

The distribution of eNOS was observed in trophoblast cells and was found to be distinct from the distribution of iNOS. It was found that eNOS was distributed in the cytoplasm and was also associated with actin filaments (Fig. 4A). No eNOS was observed at the leading edge (Fig. 4B). Significant co-localisation of eNOS and actin was observed and this co-localisation was common in both motile (Fig. 4Ai) and non-motile cells (Fig. 4Aii and iii).

iNOS and eNOS show distinct distribution patterns and do not co-localise

The distribution of the two NOS isoforms eNOS and iNOS in trophoblast cells is quite distinct, with very little co-localisation observed between the two isoforms (Fig. 5B). Quantitative co-localisation analysis revealed an overlap co-efficient of $0.16 \pm 0.04$, suggesting a poor degree of overlap in the distribu- tion of the two isoforms. iNOS in non-motile cells is mostly cytoplasmic and particulate, in motile cells iNOS is also associated with the leading edge. eNOS is distributed similarly in both motile and non-motile cells, with much of the eNOS either cytoplasmic or associated with actin (Fig. 5A). Similar results were found when specific antibodies were used to detect both iNOS and eNOS in the same cells rather than expressing the iNOS-EGFP construct (Fig. 5C and D).

Enhanced S-nitrosylation of proteins at the leading edge

Immunocytochemical staining for S-nitrosylated proteins revealed enhanced fluorescence intensity at the leading edge (Fig. 6A). Increased S-nitrosylation is indicated by the arrows and was detected in all cells that showed a distinct leading edge. The detection of S-nitrosylated proteins was confirmed in Fig. $6 \mathrm{~B}$, as reduced staining was detected in cells treated with the general nitric oxide synthase inhibitor, L-NAME.

\section{Co-localisation of protein S-nitrosylation with iNOS}

S-nitrosylated proteins were enhanced at the leading edge and were also found to co-localise with iNOS-EGFP (Fig. 7A).

A

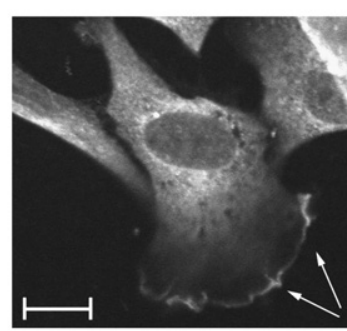

B
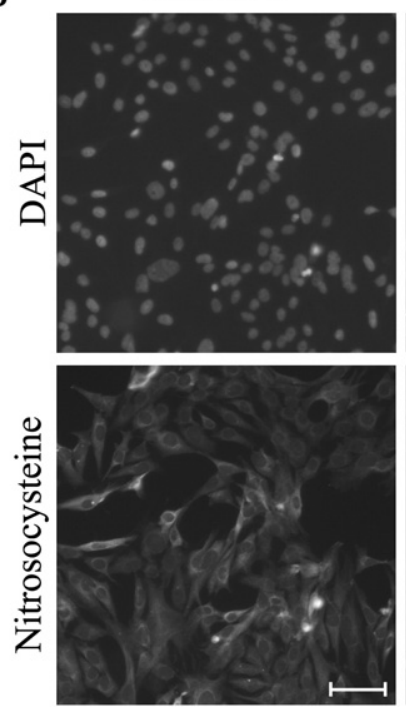

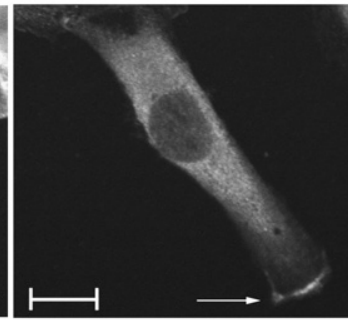

L-NAME treated
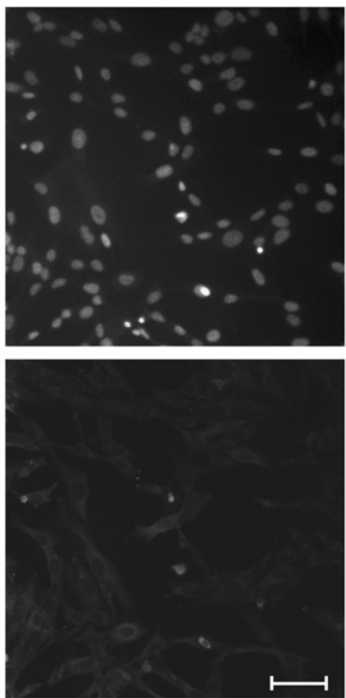

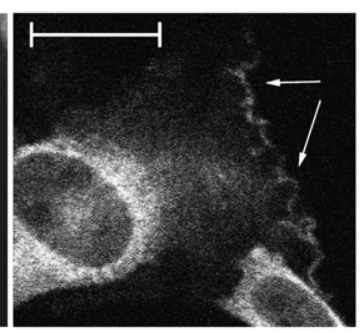

Ab control
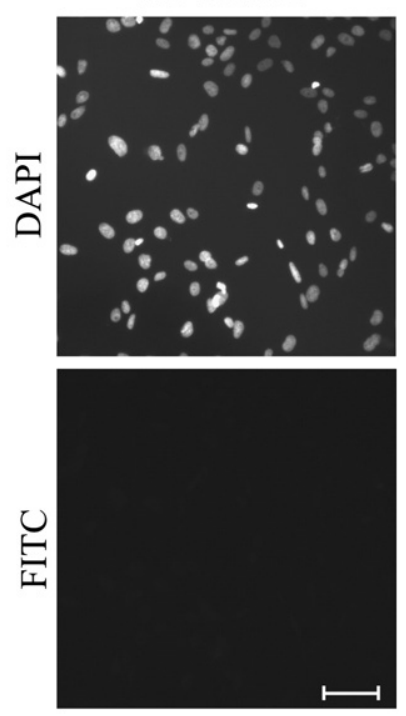

Fig. 6 - Distribution of S-nitrosylated proteins in migrating trophoblast cells. (A) Confocal microscope images of S-nitrosocysteine immunostaining in trophoblast cells. Arrows indicate the staining at the leading edge. The images shown are single z-sections representing replicate experiments and the scale bars represent $10 \mu \mathrm{m}$. (B) S-nitrosylation in a population of cells as determined by fluorescence microscopy. As a control the general nitric oxide synthase inhibitor L-NAME (5 mM) was added for $\mathbf{2 4} \mathrm{h}$. Mouse immunoglobulins were used as an antibody control. Scale bar represents $50 \mu \mathrm{m}$ and images are representative of at least 20 fields of view from three separate experiments. 
A

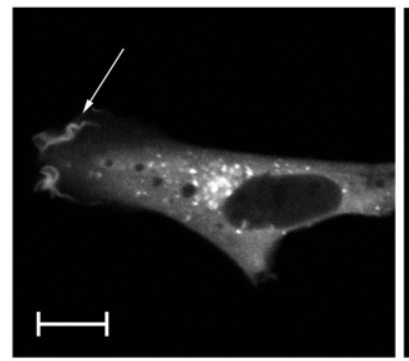

iNOS-GFP

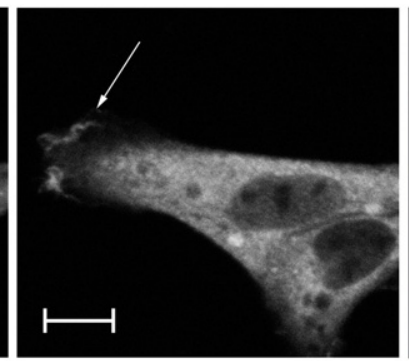

Nitrosocysteine

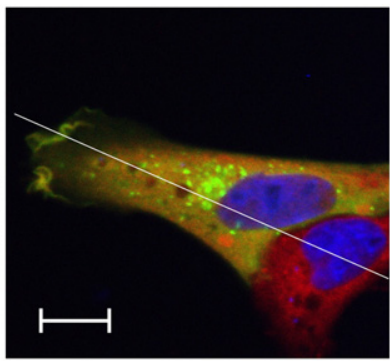

Merged

B

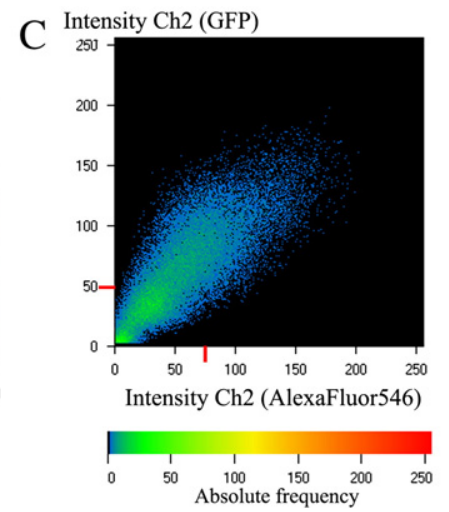

Fig. 7 - Co-localisation between iNOS and S-nitrosocysteine. (A) Confocal microscope images showing the distribution of both iNOS-EGFP (shown in green) and anti-nitrosocysteine immunostaining (shown in red) in the same cell. DAPI nuclear staining is shown in blue. The image shown is a single $z$-section, the scale bar represents $10 \mu \mathrm{m}$ and the images are representative of at least $\mathbf{4 0}$ cells in three separate experiments. Arrow indicates the leading edge and the dotted line shows the line profile that was analysed in panel B. (B) Quantitative analysis of the line from panel A showing the fluorescence intensities of iNOS-EGFP (green), S-nitrosocysteine (red) and DAPI (blue) throughout the cell. Results are representative of at least 30 cells analysed from three separate experiments. (C) Quantitative co-localisation analysis of iNOS-EGFP and S-nitrosocysteine at the leading edge. The figure is a representative of co-localisation analysis in at least 20 cells.

Quantification of the fluorescence intensity revealed a high degree of co-localisation at the leading edge and also throughout the cell (Fig. 7B). Quantitative co-localisation analysis of the leading edge region of at least 20 cells produced an overlap co-efficient of $0.81 \pm 0.05$, suggesting a high degree of overlap between iNOS and S-nitrosocysteine staining (Fig. 7C).

Co-localisation of MMP-9 with iNOS at the leading edge and the importance of iNOS for trophoblast invasion

Immunochemical staining for MMP-9 revealed staining at the leading edge of trophoblast cells (Fig. 8A), and it was found to be colocalised with iNOS (overlap co-efficient of $0.74 \pm 0.03$ ) in cells expressing the iNOS-GFP construct (Fig. 8B and C), suggesting that MMP-9 is one of the proteins at the leading edge that could be S-nitrosylated. Similar results were found in isolated primary extravillous trophoblasts (Fig. 8D), with both iNOS and MMP-9 detected at the leading edge. Treatment of cells with a general MMP inhibitor NNGH $(50 \mu \mathrm{M})$ was found to significantly $(p<0.001)$ reduce the invasion of trophoblast cells (Fig. 9). Treatment of trophoblasts with either the general NOS inhibitor L-NAME (5 mM) or the specific iNOS inhibitor 1400W $(5 \mu \mathrm{M})$ was also found to inhibit their invasion, reducing the percentage of cells crossing the filter from $48.8 \% \pm 7.5$ to $9.7 \% \pm$ $7.5(p=0.003)$ and $4.25 \% \pm 3.4(p=0.0007)$ respectively, suggesting that production of $\mathrm{NO}$, and specifically the production of NO from iNOS, plays a key role in promoting trophoblast invasion.

\section{Discussion}

Trophoblast cells, and in particular the extravillous trophoblast sub-population, are highly motile and invasive cells. During early pregnancy they invade the uterus and contribute to the remodelling of the maternal spiral arteries. This is an essential step in the establishment of a successful pregnancy as it ensures a high blood flow with lower resistance later in the pregnancy. Poor trophoblast invasion has been linked with complications of pregnancy such as pre-eclampsia [13]. The regulation of trophoblast motility and invasion is therefore an important area of investigation, although little is currently known about the molecular mechanisms involved in these processes.

Nitric oxide (NO) plays an important role in the regulation of cell migration in a variety of cell types including trophoblast cells, endothelial cells and epithelial cells [11,14-16]. Although NO plays a role in cell migration there has been little investigation 
A
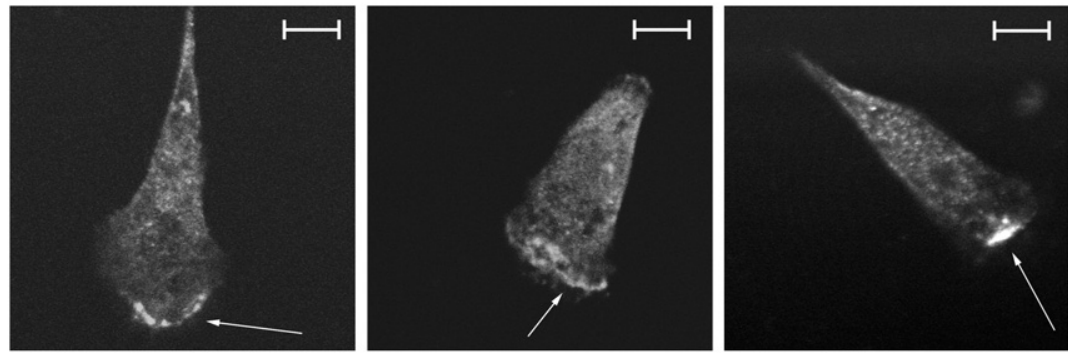

B
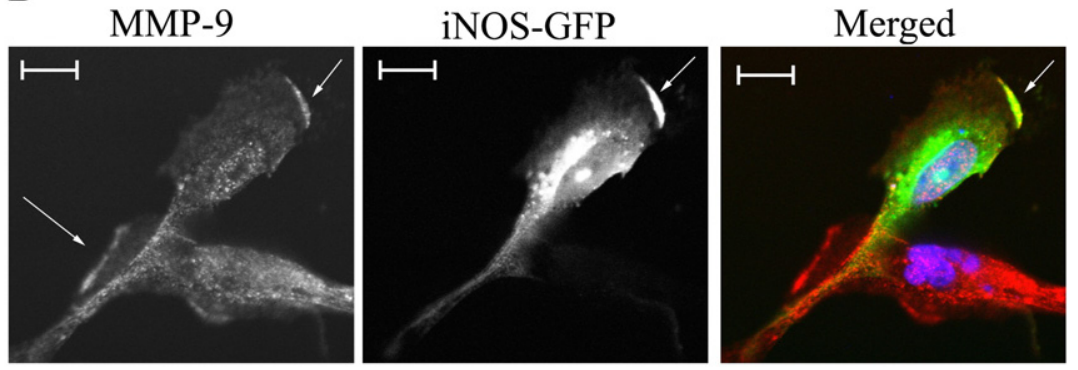

C

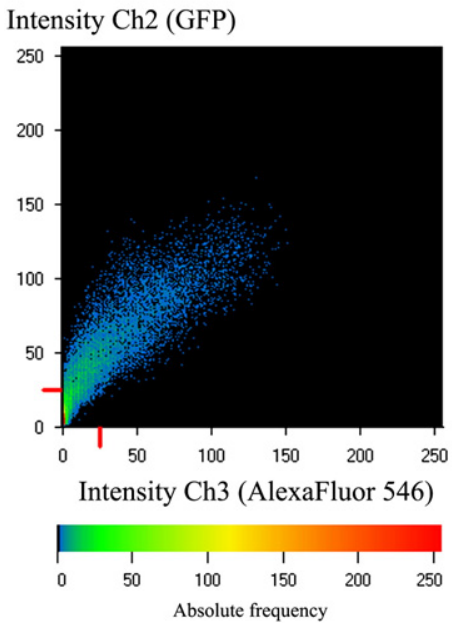

D

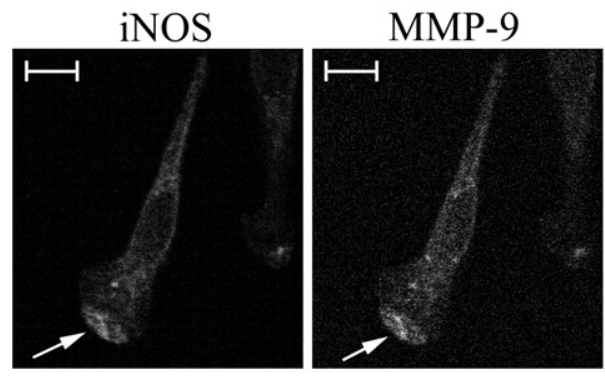

Fig. 8 - Intracellular distribution of MMP-9 in migrating trophoblasts. (A) Confocal microscope images of migrating trophoblast cells immunostained for MMP-9. Arrows indicate the leading edge of the cells. The scale bar represents $10 \mu \mathrm{m}$ and the images are representative of at least 40 cells in three separate experiments. The image shown is a single $z$-section.

(B) Co-localisation of MMP-9 and iNOS-EGFP as determined by confocal microscopy. MMP-9 is shown in red, iNOS-EGFP in green and DAPI in blue. Arrows indicate the leading edge of the cells. The image shown is a single $z$-section and is a representative of at least 30 cells from three experiments. The scale bar represents $10 \mu \mathrm{m}$. (C) Quantitative co-localisation analysis of iNOS-EGFP and MMP-9 at the leading edge. The figure is a representative of co-localisation analysis in at least 20 cells. (D) Co-localisation of MMP-9 and iNOS in primary extravillous trophoblasts as determined by immunocytochemistry and confocal microscopy.

Arrows indicate the leading edge of the cells and the region of co-localisation between MMP-9 and iNOS. The image shown is a single z-section and is a representative of at least 30 cells from three separate preparations of primary cells. The scale bar represents $10 \mu \mathrm{m}$.

into its mechanism of action. Nitric oxide can mediate its effects through a number of mechanisms including the production of cGMP from guanylate cyclase and modification of tyrosine residues in proteins. However the effects of NO on cysteine residues, a process called nitrosylation, are emerging as an important mechanism for the effects of NO and as an important signalling mechanism in cells [4]. Nitric oxide is a highly reactive and diffusible molecule, yet its effects in the cell are specific and tightly regulated. Indeed it is becoming increasingly apparent that NO production is well compartmentalised within the cell and that the nitric oxide synthase (NOS) enzymes are transported to specific regions of the cell along with the target proteins for 


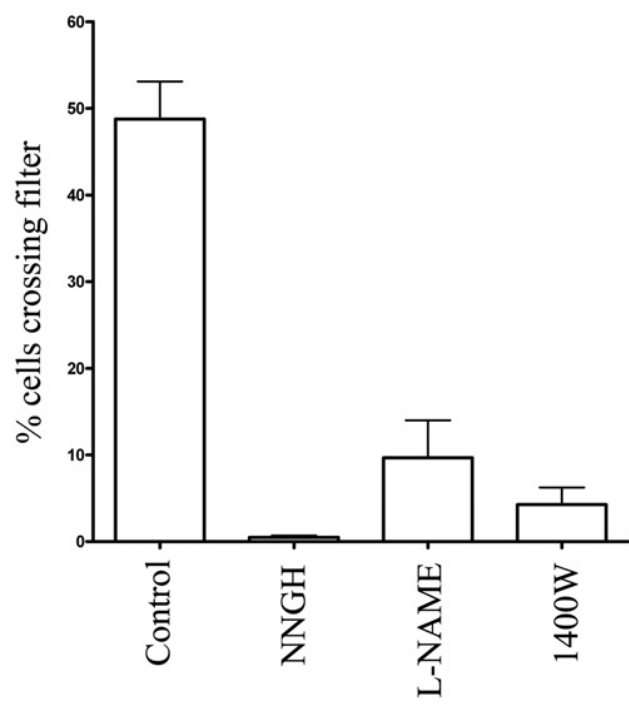

Fig. 9 - Invasion assay showing the percentage of cells crossing a filter containing Matrigel over a 24 hour period. Cells were either untreated or treated with the MMP inhibitor NNGH $(50 \mu \mathrm{M})$ or with the nitric oxide synthase inhibitors L-NAME $(5 \mathrm{mM})$ or $1400 \mathrm{~W}(5 \mu \mathrm{M})$. Results shown are mean \pm sem from triplicate experiments.

NO. The close proximity of NO production with the proteins to be nitrosylated is one factor that has been proposed to ensure the specificity of the reaction.

The intracellular distribution of eNOS and nNOS has been well studied in many cell types [22] although the intracellular distribution of iNOS has been comparatively poorly studied until recently. The few studies examining the intracellular distribution of iNOS have reported diverse findings, with iNOS reported in the cytoplasm or in vesicular or perinuclear structures $[23,24]$. In one study iNOS was identified in the Golgi [25] and in another the perinuclear distribution was attributed to aggresome formation [26]. However, the distribution of NOS isoforms, and particularly iNOS, in migrating cells has not been well studied. We have investigated the distribution of NOS isoforms in migrating trophoblast cells using high resolution confocal microscopy.

We found that iNOS was localised in several populations within trophoblast cells. An EGFP tagged iNOS was expressed in trophoblasts and was found in the cytoplasm, in the form of iNOS particles, or aggresomes, and was located at the leading edge of migrating cells. Similar results were obtained when antibodies were used to detect endogenous iNOS in migrating trophoblasts. It has been reported that iNOS plays a role in migrating epithelial cells and that it may act as a switch from a stationary to a locomotive phenotype in these cells [14]. In this study the epithelial cells did not express iNOS until wounds were inflicted in the cells. Following the creation of wounds, cells nearest the edge of the wound demonstrated significantly increased iNOS expression and became more motile. However high resolution images were not obtained in this study and it was not possible to determine the intracellular distribution of iNOS in these cells, although NADPH diaphorase activity assays, an indirect assay for general NOS activity, did suggest that there may be increased NOS expression in the front region of the cell.

In this study we report the distribution of iNOS to the leading edge of migrating trophoblast cells. Quantification of fluorescence intensity revealed that there was an almost 4-fold increase in the iNOS expression at the leading edge compared to the expression of iNOS in the cytoplasm behind the leading edge or in the trailing edge. There was greater fluorescence intensity at the leading edge than in any other region of the cell, with the exception of the iNOS particles or aggresomes. To determine that proteins at the leading edge are being specifically enriched in this region cells were labelled with a CellTracker dye, a non-specific protein reactive fluorescent dye. We found that there was no increased fluorescence of the CellTracker dye at the leading edge despite a significant peak of iNOS-EGFP fluorescence intensity at this site, suggesting that iNOS is specifically enriched at the leading edge.

During cell migration the leading edge of a cell demonstrates membrane protrusion, adhesion site formation and the generation of force. The protrusion of the plasma membrane and the formation of the lamellipodium, are driven by polymerisation of actin at the cell front. Since the leading edge of migrating cells is a site of significant actin polymerisation we investigated the distribution of iNOS in relation to the cytoskeleton components actin and tubulin. We found that the distribution of iNOS shows substantial overlap with the region of extensive actin staining. In most cases actin and iNOS overlapped or colocalised, although in some cases it appeared that the iNOS was distributed slightly ahead of the main area of actin polymerisation. By contrast there was no significant correlation between iNOS distribution at the leading edge and tubulin staining. To study this process in more detail, the distribution of iNOS was examined in live cells using an iNOScitrine construct. In live migrating cells iNOS was rapidly redistributed around the leading edge, apparently co-incident with the formation of membrane protrusions. This dynamic re-distribution of iNOS at the leading edge suggests that iNOS is likely to play an important role in cell migration and is not merely passively accumulating at the leading edge.

We examined the distribution of iNOS in non-migrating cells using either serum-starved or confluent cells. In both cases iNOS was found pre-dominantly in the cytoplasm or in the form of iNOS particles/aggresomes. Quantification of the fluorescence intensity across the cell showed that there was no increased iNOS fluorescence at the edges of non-migrating cells, suggesting that the iNOS localisation at the leading edge was indeed a property of the cell migration and is not observed in non-motile cells.

Trophoblasts express both eNOS and iNOS and the production of NO from both these isoforms is likely to be important. It has been previously reported that eNOS localises to the leading edge of migrating HeLa and endothelial cells [27]. In trophoblasts, we demonstrate that eNOS is distributed mostly in the cytoplasm with none found at the leading edge. In addition, in many cells eNOS can be found distributed in or along what appear to be filaments. Staining of cells with a fluorescent phalloidin stain revealed that they are likely to be actin filaments and quantitative co-localisation analysis showed that there is a high degree of co-localisation between eNOS and actin. This actin is distinct from the actin that 
undergoes extensive polymerisation at the leading edge and is more characteristic of stress fibres. There have been some reports that eNOS can associate with actin [28] and that this association might increase its activity, suggesting that eNOS may be more active in these regions.

There is good evidence that the spatial distribution of NOS isoforms plays an important role in regulating the effects of NO [22] and that this compartmentalisation of NO production allows for distinct, and in some cases antagonistic, effects of NO in the same cell [29]. We examined the distribution of iNOS and eNOS in the same cells and found that there is strict separation of the two isoforms, with no significant co-localisation. This suggests that NO production at the leading edge is limited to NO produced from iNOS. Interestingly in cells without a distinct leading edge there is no iNOS detected around the edges of the cell but eNOS still appears to be aligned along actin filaments, suggesting that if eNOS has a role in regulating trophoblast migration it is through a mechanism distinct from that of iNOS.

Localisation of NOS has recently been shown to determine the site of protein S-nitrosylation. Targeting of eNOS to the Golgi leads to enhanced overall Golgi protein S-nitrosylation and to the S-nitrosylation of Golgi specific proteins [21]. Since iNOS is localised to the leading edge in migrating trophoblast cells we investigated the distribution of S-nitrosylated proteins in these cells. Migrating cells showed enhanced S-nitrosocysteine staining at the leading edge, suggesting that NO produced at this site leads to significant protein S-nitrosylation. The distribution of S-nitrosylated proteins in cells expressing iNOS-EGFP showed that S-nitrosylated proteins showed similar distribution to iNOS, with the exception of the iNOS particles or aggresomes which showed no significant correlation with S-nitrosocysteine staining. This finding suggests that these iNOS aggresomes are not sites of significant protein S-nitrosylation. Quantitative colocalisation analysis of the leading edge revealed that there is significant co-localisation between iNOS and S-nitrosylated proteins at this site, demonstrating that the presence of iNOS, and therefore the production of $\mathrm{NO}$ at the leading edge may be responsible for the S-nitrosylation of proteins in this region. It has recently been shown that an important regulator of NO production, dimethylarginine dimethylaminohydrolase (DDAH) is located at the leading edge of migrating endothelial cells [30]. DDAH metabolises ADMA, an endogenous inhibitor of nitric oxide synthesis, and its location at the leading edge suggests that this would be a site of increased NO production and further supports the hypothesis that NO production at the leading edge of migrating cells plays an important role in regulating either cell motility or cell invasion.

There are many proteins at the leading edge that are involved in regulating these processes and determining which proteins are regulated by NO would improve our understanding of the role of NO in cell migration and invasion. One possible way to identify which proteins are modified by NO is to use the biotin-switch technique [31] in combination with standard proteomic techniques. An initial proteomic screen (data not shown) suggested that one of the matrix metalloproteinases, MMP-9, is nitrosylated in trophoblast cells. Nitric oxide is known to increase the expression and activity of matrix metalloproteinases in many cell types [32-34], including trophoblasts [35]. It has also been shown that MMP-9 is nitrosylated [36], and that this nitrosylation occurs in the cysteine located in the cysteine switch domain of the protein which is required for activation of the enzyme. Nitrosylation of this cysteine can then lead to its oxidation to sulfinic or sulfonic acid derivatives. These further modifications are irreversible and can lead to the permanent activation of MMP-9 [36].

In invading cells it is logical to suppose that MMPs will be secreted from the leading edge in order to degrade the matrix ahead of the cell. Since iNOS is located at the leading edge it is possible that it might interact with MMP-9, increasing its activity and enhancing the degradation of the extracellular matrix. To investigate this hypothesis we examined the distribution of MMP-9 in trophoblast cells and found that there was significant staining for MMP-9 around the leading edge of migrating cells. We also examined the distribution of MMP-9 in cells expressing the iNOS-EGFP construct and found a significant degree of co-localisation between the two proteins, supporting the idea that they might interact in the cell. Colocalisation between iNOS and MMP-9 was also observed in primary extravillous trophoblast cells using antibodies against the two proteins. Although this co-localisation at the leading edge supports the hypothesis that MMP-9 is nitrosylated it is also possible that NO may be having other effects on MMP-9, for example by promoting its secretion.

We also performed functional studies to investigate the regulation of trophoblast invasion by NO. Using invasion assays we demonstrated that, as would be expected, inhibition of MMP activity with a broad spectrum MMP inhibitor almost completely inhibited invasion through a Matrigel matrix. We also found a significant reduction in the invasion of these cells when they were treated with either a general NOS inhibitor or with an iNOS specific inhibitor, suggesting that production of NO from iNOS plays an important role in the regulation of trophoblast invasion.

\section{Conclusions}

We conclude that iNOS localises to the leading edge and promotes trophoblast invasion. This may be achieved by the interaction of iNOS with MMP-9 at the leading edge, possibly leading to the nitrosylation of MMP-9, which is thought to lead to increased activity. Trophoblast migration and invasion are essential processes for the establishment of a successful pregnancy, with poor trophoblast invasion linked to complications of pregnancy such as pre-eclampsia and intra-uterine growth restriction. In this study we show that the intracellular distribution of iNOS and the specific S-nitrosylation of proteins at the leading edge may play an important role in regulating these processes.

\section{R E F E R E N C E S}

[1] T.M. Greco, R. Hodara, I. Parastatidis, H.F. Heijnen, M.K. Dennehy, D.C. Liebler, H. Ischiropoulos, Identification of S-nitrosylation motifs by site-specific mapping of the $\mathrm{S}$-nitrosocysteine proteome in human vascular smooth muscle cells, Proc. Natl. Acad. Sci. U. S. A. 103 (2006) 7420-7425.

[2] D.T. Hess, A. Matsumoto, S.O. Kim, H.E. Marshall, J.S. Stamler, Protein S-nitrosylation: purview and parameters, Nat. Rev. Mol. Cell. Biol. 6 (2005) 150-166. 
[3] J.B. Mannick, A. Hausladen, L. Liu, D.T. Hess, M. Zeng, Q.X. Miao, L.S. Kane, A.J. Gow, J.S. Stamler, Fas-induced caspase denitrosylation, Science 284 (1999) 651-654.

[4] J.S. Stamler, S. Lamas, F.C. Fang, Nitrosylation. the prototypic redox-based signaling mechanism, Cell 106 (2001) 675-683.

[5] R. Pijnenborg, L. Vercruysse, M. Hanssens, The uterine spiral arteries in human pregnancy: facts and controversies, Placenta 27 (2006) 939-958.

[6] B. Schiessl, I. Mylonas, P. Hantschmann, C. Kuhn, S. Schulze, S. Kunze, K. Friese, U. Jeschke, Expression of endothelial NO synthase, inducible NO synthase and estrogen receptors alpha and beta in placental tissue of normal, preeclamptic, and intrauterine growth restricted pregnancies, J. Histochem. Cytochem. (2005).

[7] D. Martin, K.P. Conrad, Expression of endothelial nitric oxide synthase by extravillous trophoblast cells in the human placenta, Placenta 21 (2000) 23-31.

[8] P.R. Dash, G.S. Whitley, L.J. Ayling, A.P. Johnstone, J.E. Cartwright, Trophoblast apoptosis is inhibited by hepatocyte growth factor through the Akt and beta-catenin mediated up-regulation of inducible nitric oxide synthase, Cel. Signal. 17 (2005) 571-580.

[9] P.R. Dash, J.E. Cartwright, P.N. Baker, A.P. Johnstone, G.S. Whitley, Nitric oxide protects human extravillous trophoblast cells from apoptosis by a cyclic GMP-dependent mechanism and independently of caspase 3 nitrosylation, Exp. Cell Res. 287 (2003) 314-324.

[10] P.R. Dash, J.E. Cartwright, G.S. Whitley, Nitric oxide inhibits polyamine-induced apoptosis in the human extravillous trophoblast cell line SGHPL-4, Hum. Reprod. 18 (2003) 959-968.

[11] J.E. Cartwright, D.P. Holden, G.S. Whitley, Hepatocyte growth factor regulates human trophoblast motility and invasion: a role for nitric oxide, British. J. Pharmacol. 128 (1999) 181-189.

[12] J.E. Cartwright, W.K. Tse, G.S. Whitley, Hepatocyte growth factor induced human trophoblast motility involves phosphatidylinositol-3-kinase, mitogen-activated protein kinase, and inducible nitric oxide synthase, Exper. Cell. Res. 279 (2002) 219-226.

[13] P. Kaufmann, S. Black, B. Huppertz, Endovascular trophoblast invasion: implications for the pathogenesis of intrauterine growth retardation and preeclampsia, Biol. Reprod. 69 (2003) $1-7$.

[14] E. Noiri, T. Peresleni, N. Srivastava, P. Weber, W.F. Bahou, N. Peunova, M.S. Goligorsky, Nitric oxide is necessary for a switch from stationary to locomoting phenotype in epithelial cells, Amer. J. Phys. 270 (1996) C794-C802.

[15] K. Purdie, G.S.J. Whitley, A.P. Johnstone, J.E. Cartwright, Hepatocyte growth factor-induced endothelial cell motility is mediated by upregulation of inducible nitric oxide synthase, Circ. Res. 54 (2002) 659-668.

[16] D. Giordano, D.M. Magaletti, E.A. Clark, Nitric oxide and cGMP protein kinase (cGK) regulate dendritic-cell migration toward the lymph-node-directing chemokine CCL19, Blood 107 (2006) 1537-1545.

[17] M.Y. Choy, I.T. Manyonda, The phagocytic activity of human first trimester extravillous trophoblast, Human Reproduct. 13 (1998) 2941-2949.

[18] K.T. Shiverick, A. King, H.G. Frank, G.S.J. Whitley, J.E. Cartwright, H. Schneider, Cell culture models of human trophoblast II: trophoblast cell lines - a workshop report, Placenta 22 (2001) S104-S106.

[19] J.E. Cartwright, L.C. Kenny, P.R. Dash, J.D. Aplin, P.N. Baker, G. S.J. Whitley, Trophoblast invasion of spiral arteries: a novel in vitro model, Placenta 23 (2002) 232-235.

[20] A. Tarrade, R. Lai Kuen, A. Malassine, V. Tricottet, P. Blain, M. Vidaud, D. Evain-Brion, Characterization of human villous and extravillous trophoblasts isolated from first trimester placenta, Laboratory. Invest. 81 (2001) 1199-1211.
[21] Y. Iwakiri, A. Satoh, S. Chatterjee, D.K. Toomre, C.M. Chalouni, D. Fulton, R.J. Groszmann, V.H. Shah, W.C. Sessa, Nitric oxide synthase generates nitric oxide locally to regulate compartmentalized protein S-nitrosylation and protein trafficking, Proc. Natl. Acad. Sci. U. S. A. 103 (2006) 19777-19782.

[22] S. Oess, A. Icking, D. Fulton, R. Govers, W. Muller-Esterl, Subcellular targeting and trafficking of nitric oxide synthases, Biochem. J. 396 (2006) 401-409.

[23] Y. Vodovotz, D. Russell, Q.W. Xie, C. Bogdan, C. Nathan, Vesicle membrane association of nitric oxide synthase in primary mouse macrophages, J. Immunol. 154 (1995) 2914-2925.

[24] M.A. Wheeler, S.D. Smith, G. Garcia-Cardena, C.F. Nathan, R.M. Weiss, W.C. Sessa, Bacterial infection induces nitric oxide synthase in human neutrophils, J. Clin. Invest. 99 (1997) 110-116.

[25] J.L. Webb, M.W. Harvey, D.W. Holden, T.J. Evans, Macrophage nitric oxide synthase associates with cortical actin but is not recruited to phagosomes, Infect. Immun. 69 (2001) 6391-6400.

[26] K.E. Kolodziejska, A.R. Burns, R.H. Moore, D.L. Stenoien, N.T. Eissa, Regulation of inducible nitric oxide synthase by aggresome formation, Proc. Natl. Acad. Sci. U. S. A. 102 (2005) 4854-4859.

[27] S. Bulotta, A. Cerullo, R. Barsacchi, C.D. Palma, D. Rotiroti, E. Clementi, N. Borgese, Endothelial nitric oxide synthase is segregated from caveolin-1 and localizes to the leading edge of migrating cells, Exp. Cell Res. 312 (2006) 877-889.

[28] Y. Su, S. Edwards-Bennett, M.R. Bubb, E.R. Block, Regulation of endothelial nitric oxide synthase by the actin cytoskeleton, Am. J. Physiol. Cell. Physiol. 284 (2003) C1542-C1549.

[29] L.A. Barouch, R.W. Harrison, M.W. Skaf, G.O. Rosas, T.P. Cappola, Z.A. Kobeissi, I.A. Hobai, C.A. Lemmon, A.L. Burnett, B. O’Rourke, E.R. Rodriguez, P.L. Huang, J.A. Lima, D.E. Berkowitz, J.M. Hare, Nitric oxide regulates the heart by spatial confinement of nitric oxide synthase isoforms, Nature 416 (2002) 337-339.

[30] B. Wojciak-Stothard, B. Torondel, L.Y. Tsang, I. Fleming, B. Fisslthaler, J.M. Leiper, P. Vallance, The ADMA/DDAH pathway is a critical regulator of endothelial cell motility, J. Cell. Sci. 120 (2007) 929-942.

[31] S.R. Jaffrey, H. Erdjument-Bromage, C.D. Ferris, P. Tempst, S.H. Snyder, Protein S-nitrosylation: a physiological signal for neuronal nitric oxide, Nature Cell Biol. 3 (2001) 193-197.

[32] E. Lopez-Rivera, T.R. Lizarbe, M. Martinez-Moreno, J.M. Lopez-Novoa, A. Rodriguez-Barbero, J. Rodrigo, A.P. Fernandez, A. Alvarez-Barrientos, S. Lamas, C. Zaragoza, Matrix metalloproteinase 13 mediates nitric oxide activation of endothelial cell migration, Proc. Natl. Acad. Sci. U. S. A. 102 (2005) 3685-3690.

[33] P.F. Bove, U.V. Wesley, A.K. Greul, M. Hristova, W.R. Dostmann, A. van der Vliet, Nitric oxide promotes airway epithelial wound repair through enhanced activation of MMP-9, Am. J. Respir. Cell. Mol. Biol. 36 (2007) 138-146.

[34] C. Zaragoza, E. Lopez-Rivera, C. Garcia-Rama, M. Saura, A. Martinez-Ruiz, T.R. Lizarbe, F. Martin-de-Lara, S. Lamas, Cbfa-1 mediates nitric oxide regulation of MMP-13 in osteoblasts, J. Cell. Sci. 119 (2006) 1896-1902.

[35] V. Novaro, A. Colman-Lerner, F.V. Ortega, A. Jawerbaum, D. Paz, F. Lo Nostro, C. Pustovrh, M.F. Gimeno, E. Gonzalez, Regulation of metalloproteinases by nitric oxide in human trophoblast cells in culture, Reprod. Fertil. Dev. 13 (2001) 411-420.

[36] Z. Gu, M. Kaul, B. Yan, S.J. Kridel, J. Cui, A. Strongin, J.W. Smith, R.C. Liddington, S.A. Lipton, S-nitrosylation of matrix metalloproteinases: signaling pathway to neuronal cell death, Science 297 (2002) 1186-1190. 